\title{
Sozialpädagogik als disziplinäre Grenzgänger*in
}

\author{
Bettina Hünersdorf
}

Online publiziert: 17. Juli 2020

(C) Der/die Autor(en) 2020

Zusammenfassung Sozialpädagogik als disziplinäre Grenzgänger*in nimmt die disziplinäre Form der Sozialpädagogik in den Anfängen ihrer Versozialwissenschaftlichung in den Blick. Dabei wird eine Lesart von Aloys Fischers Sozialpädagogik entwickelt, die mit ihm und über ihn hinaus gehend die These begründet, dass die Sozialpädagogik sich durch eine doppelte Offenheit kennzeichne: zum einen die Offenheit gegenüber anderen sozialwissenschaftlichen Disziplinen wie der Soziologie und der Psychologie, welche zur interdisziplinären Konstitution der Sozialpädagogik beitrage, zum anderen die Offenheit gegenüber der professionellen Praxis. Erst durch diese doppelt miteinander verschränkte Relationierung der Sozialpädagogik ergibt sich ihre spezifische, sich gegenüber anderen Sozialwissenschaften unterscheidende Gestalt. Mit der ,deskriptiven“ Sozialpädagogik gibt es Anknüpfungspunkte für eine erkenntnistheoretische und methodologische Grundlegung, die durch sinnliche Erkenntnisbildung vermag zwischen einer sozialwissenschaftlich generierten Theorie der Sozialpädagogik und einer professionellen Praxis der Sozialpädagogik zu vermitteln. Dadurch bekommt die Sozialpädagogik als Disziplin eine performative (zukunfts-)offene Form, in welcher der Bruch zwischen sozialpädagogischer Theorie und professioneller Praxis nicht als Problem, sondern als Möglichkeit wahrgenommen wird, sich (un-)abhängig voneinander zu konstituieren.

Schlüsselwörter Sozialpädagogische Theorie · Sozialpädagogik als Disziplin · Sozialpädagogik als Profession · Deskriptive Sozialpädagogik

Prof. Dr. B. Hünersdorf $(\bowtie)$

Institut für Pädagogik, Phil Fak. III., Martin-Luther-Universität Halle-Wittenberg, 06099 Halle, Deutschland

E-Mail: bettina.huenersdorf@paedagogik.uni-halle.de 


\section{Social pedagogy as a disciplinary cross-border commuter}

Abstract Social pedagogy as a disciplinary cross-border commuter looks at the disciplinary form of social pedagogy in the early stages of its beginnings as a social science. The development of a new reading of and beyond Aloys Fischer's social pedagogy shows that social pedagogy is characterized by a double openness: on the one hand, the openness to various other social science disciplines such as sociology and psychology, which contribute to the interdisciplinary constitution of social pedagogy, on the other hand the openness of the professional practice. It is only through this double interrelation of social pedagogy that it differs in its form from sociology and psychology. Descriptive social pedagogy provides a starting point for an epistemological and methodological foundation that, through the formation of sensual knowledge, can mediate between a social science-generated theory of social pedagogy and the social practice of social pedagogy. This gives social pedagogy as a discipline a performative contingent form, in which the disruption between social pedagogical theory and professional practice is not perceived as a problem, but rather as an opportunity to be constituted (in-)dependently of one another.

Keywords Theory of social pedagogy $\cdot$ Social pedagogy as disciplin · Socialpedagogy as profession · Descriptive socialpedagogy

\section{Einleitung}

Für die Disziplinenentwicklung spielen Theorien und Methoden wie institutionelle Möglichkeiten der Nachwuchsrekrutierung eine zentrale Rolle (Stichweh 2017, S. $181 \mathrm{f}$.). Die Bildung wissenschaftlicher Disziplinen, die auf die Mitte des 18. Jahrhunderts (1750-1870) datiert werden kann (vgl. Stichweh 2017), ging der Entstehung der Sozialpädagogik voraus. Sozialpädagogik gab es zu der Zeit nur als Theoriefrage, nicht aber als Methodenfrage und schon gar nicht in Form einer Institutionalisierung von Professuren an Universitäten. Im Selbstverständnis der Sozialpädagogik wird häufig darauf verwiesen, dass diese eine eher offene Disziplin sei, die sich seit den 1970er Jahren institutionalisiert und sich im Laufe der Jahre zunehmend etabliert habe. Dieses könne man an der Theoriebildung, dem Umfang empirischer Forschung, sowie der Nachwuchsrekrutierung erkennen (vgl. Thole 1994). Trotz der Etablierung sei die Sozialpädagogik aber in ihren Grenzen offen (vgl. Thole und Galuske 2003; Cloos et al. 2020).

Ich möchte in meinen folgenden Ausführungen zeigen, dass für die Sozialpädagogik als disziplinäre Praxis eine doppelte Offenheit konstitutiv ist. Diese bezieht sich, so meine These, zum einen auf die Offenheit ihrer Disziplingrenzen zu anderen Disziplinen hin. Im Fokus steht dann die Frage nach der interdisziplinären Konstitution der Sozialpädagogik. Dabei werden Diskurse aus anderen Diszipli- 
nen aufgegriffen, aber diese werden transformiert (vgl. Dollinger 2006) ${ }^{1}$. Ich werde entsprechend darstellen, wie Sozialpädagogik als Disziplin disziplinäre Grenzarbeit (vgl. Kessl und Maurer 2010) gegenüber anderen Disziplinen leistet, um sich trotz aller Durchdringung durch andere Disziplinen bzw. der Entgrenzung gegenüber den Sozialwissenschaften (Stadler-Altmann und Gross 2019, S. 10) als eigene Disziplin darstellen zu können. Dabei folgt die Sozialpädagogik als disziplinäre Praxis einer ,pragmatischen Übersetzung“ (Renn 2014, S. 39). Das tertium comparationis ermöglicht den Übergang zwischen den Sozialwissenschaften und der sich versozialwissenschaftlichenden Sozialpädagogik als implizite kulturelle Form der Disziplin, die durch die Generierung eigener Theorien und Methoden in Abgrenzung zu denen der anderen Sozialwissenschaften explizit wird (Renn 2014, S. 40). Dabei ist die eigene (inter-)disziplinäre Form der Sozialpädagogik eng verknüpft mit einer anderen Offenheit, der Bezugnahme auf das Theorie-Praxis-Verhältnis in der Sozialpädagogik (vgl. Sandermann und Neumann 2018), was auch als Relation von Disziplin und Profession verhandelt wird (vgl. Kessl 2020). Die Sozialpädagogik unterscheidet als (inter-)disziplinäre und professionelle Praxis implizites kulturelles sozialpädagogisches Wissen mit einem darin eingelagerten „Geltungsmodus der unthematischen Gewissheit von expliziten kulturellen Wissenssystemen und ihrer Legitimationsform“ (Renn 2014, S. 42). Dabei muss die Frage nach der Form und Funktion des impliziten sozialpädagogischen Wissens gestellt werden. Darüber hinaus wird der Blick auf die expliziten kulturellen Wertsphären der Sozialpädagogik gerichtet, die als Pfade der Rationalisierung des sozialpädagogischen Wissens rekonstruiert werden können (vgl. Renn 2014). „Zur Rekonstruktionen der Formen und Wege und Folgen der Explikation und der strukturbildenden Reflexivierung [von sozialpädagogischem Wissen] gehören sowohl medientheoretische Untersuchungen [...] als auch die Analyse der pragmatischen Formen der Umsetzung expliziter Regeln und abstrakter Strukturen in konkreten Handlungen“ (Renn 2014, S. 43). In den medientheoretischen Untersuchungen werden sozialpädagogische Erkenntnisprozesse als kulturelle Praxen der Sichtbarkeit und der Sagbarkeit rekonstruiert. In der Analyse der pragmatischen Formen der Umsetzung expliziter Regeln, differenzieren diese sich zwischen professionellem Handeln und sozialpädagogischer Theoriebildung aus ${ }^{2}$.

\footnotetext{
${ }^{1}$ Bernd Dollinger (2006) zeigt auf, dass die Sozialpädagogik nicht nur reaktiv auf die in der sozialen Frage gipfelnden gesellschaftlichen Krisen reagiere, sondern diese vielmehr aktiv „durch sozialpädagogische Ausführungen an Krisendiskursen partizipieren, um sich selbst zu positionieren" (Dollinger 2006, S. 18). Das bedeutet aber auch, dass die Problembearbeitung der Sozialpädagogik der Gestalt ihres ,Gegenstandes “ folgt, welcher eben nicht in eine gesellschaftswissenschaftliche Perspektive aufgeht, sondern sich eine, wie ich an Fischer exemplarisch zeigen werde, ,Gestaltungsaufgabe ' zuschreibt. Diese zeigt sich nicht nur als praktische Aufgabe, sondern zugleich als gestaltgebend für die wissenschaftliche Sozialpädagogik, somit für ihre Disziplin.

2 Die Untersuchung der disziplinären Praxis muss, wenn sie „objektivistische (assimilative) Projektionen und die verzeichnende Subsumtion kultureller Lebensformen unter ihre[n] eigenen analytischen Kategorien vermeiden will, [sozialpädagogische; B.H.] Kategorien in praktischen Kontexten der Untersuchung erfahrungsoffen auf den Prüfstand stellen; und sie muss dabei notwendig in Kauf nehmen, dass der Gegenstand, den sie untersuchen will, sich selbst im Prozess der Untersuchung verwandelt, weil die praktische Forschungsarbeit und -interaktion ihrerseits zur Kontextbedingung im pragmatischen Forschungsfeld wird und damit zu einem Reflexionsanstoß für ,das Erforschte" selbst“ (Renn 2014, S. 44).
} 
Um die These der doppelten Offenheit der Sozialpädagogik als Disziplin zu verfolgen, werde ich einen Blick auf die Diskursgeschichte der Sozialpädagogik in der Zeit lenken, in der sich die Sozialpädagogik zwar noch nicht als Disziplin an den Universitäten entwickelt hatte, aber einen ordnenden theoretischen Sinnzusammenhang bildete (Winkler 1988, S. 59). Dabei interessiert mich, wie sich im ersten Drittel des 20. Jahrhunderts die disziplinären Bezugsysteme der Erziehungswissenschaft wie auch der Sozialpädagogik veränderten. Ich werde aufzeigen, wie sich durch die Verschiebungen der Referenzdisziplinen Diskurse der Sozialpädagogik transformier(t)en. Dabei werde ich den Übergang von einer philosophisch bestimmten Sozialpädagogik zu einer sich versozialwissenschaftlichenden und vergeisteswissenschaftlichenden Sozialpädagogik exemplarisch an Aloys Fischer darstellen. Aloys Fischer erscheint mir insofern von besonderem Interesse, da mit ihm deutlich wird, wie durch die Versozialwissenschaftlichung der Sozialpädagogik, die vorher mit Paul Natorp entworfene transzendental-kritische Grundlegung der Sozialpädagogik, sich im Verhältnis von Individuum - Gemeinschaft und Gesellschaft durch sozialwissenschaftliche ,Durchdringung , entleerte‘. Stattdessen entstand eine spezifische Form der akademischen Verberuflichung der (Sozial-)Pädagogik, die sich neben einer sozialwissenschaftlich geprägten Ausbildung durch eine deskriptive (Sozial-)Pädagogik begründete ${ }^{3}$. Ich möchte aufzeigen, dass diese Transformation möglich wurde, weil durch die (inter-)disziplinäre Orientierung, die sich als Versozialwissenschaftlichung der Sozialpädagogik darstellt(e), sich diese entäußerte und in eine sozialpädagogische Tatsachenforschung ${ }^{4}$ transformierte, die Grundlage einer akademischen Verberuflichung der Sozialpädagogik geworden ist.

\section{Zur erkenntnispolitischen Position der (Sozial-)pädagogik Aloys Fischers im Kontext ihrer Versozialwissenschaftlichung}

A. Fischer folgte einer Entwicklung der Entstehung der Sozialwissenschaften an den Universitäten, die gegenüber der Philosophie zunehmend an Bedeutung gewonnen haben (Niemeyer 1998, S. 107; Reyer 2002). Sowohl die Psychologie in ihrer

\footnotetext{
3 Damit unterscheide ich mich von Jürgen Reyers (2000, 2002) Zugang, der zwischen dem theoretischen Diskurs der Sozialpädagogik und dem verberuflichten Diskurs der Sozialpädagogik einen Bruch sieht, so als ob das eine mit dem anderen kaum in Verbindung steht. Dabei übersieht er A. Fischers Beitrag zur akademischen Verberuflichung der Sozialpädagogik. Dieser erscheint mir insofern interessant, da die ,Schelte', dass der Bezug auf die Profession auf Kosten der Disziplinenbildung gegangen sei (vgl. Münchmeier 2004, S. 22; Lüders 1989, S. 198 ff.) zu kurz greift.

4 A. Fischer greift den Begriff der Tatsachenforschung auf, welcher methodologisch einer als positivistisch verstehenden Sozialwissenschaft zuzuschreiben ist (Bonß 1983, S. 59), wendet dieses aber phänomenologisch. Dadurch kann er sich sowohl gegenüber der geisteswissenschaftlichen Pädagogik Wilhelm Diltheys als auch gegenüber einer positivistischen Pädagogik Meumanns abgrenzen. Ich werde im nächsten Kapitel genauer darauf eingehen, wie er dieses vollzieht und was dadurch (v)er(-un-)möglicht wird.
} 
Differenzierung als auch die Soziologie ${ }^{5}$ spielten aufgrund ihres begrifflichen Fundaments, wie auch durch empirische Forschung, für A. Fischer eine zentrale Rolle. Um A. Fischers Position im Kontext von Soziologie und Psychologie historisch besser bestimmen zu können, möchte ich zunächst kurz auf die Situation in der Soziologie und Psychologie eingehen und darauffolgend aufzeigen wie A. Fischer sich dazu ins Verhältnis setzte.

$\mathrm{Zu}$ Beginn des 20. Jahrhunderts ist in der Soziologie wie in der Psychologie eine Auseinandersetzung darüber erkennbar, ob diese eher philosophisch begründete Disziplinen seien oder sich von der Philosophie lösen sollten ${ }^{6}$. Dieser Streit wurde zwischen Vertretern wie Ferdinand Tönnies aber auch Georg Simmel, Rudolf Goldscheidt und Max Weber ${ }^{7}$ vehement ausgefochten. Positivistisch ausgerichtete Vertreter wie M. Weber setzten sich für eine wertneutrale Soziologie ein. Diese versprach, die Unabhängigkeit der Soziologie als Disziplin zu ermöglichen. Dennoch setzte sich diese Position gegenüber einer philosophisch begründeten ,reinen " Soziologie, wie sie von F. Tönnies ${ }^{8}$ und G. Simmel vertreten wurde, nicht durch (Sala 2017, S. 566f.). Sie verstanden Soziologie als Methode geschichtswissenschaftlicher Forschung (Simmel 1983 [1908])9 , was bedeutete, dass die Soziologie gegenüber der Philosophie ,nur relativ eigenständig“ (Korte 2004, S. 88) wäre ${ }^{10}$. Durch diese Ausrichtung wurde auch das Diktum der Wertneutralität in Frage gestellt (vgl. Korte 2004).

Zugleich gab es in der Deutschen Gesellschaft für Soziologie (DGS) die Fronstellung gegenüber nationalökonomisch ausgerichteten Staatswissenschaften, deren

\footnotetext{
5 Auch die Institutionalisierung der Soziologie als universitäre Disziplin in Deutschland war zu Beginn des 20. Jahrhunderts ein fragiles Projekt. Vielmehr ging es zu dieser Zeit auch darum, methodologische Ansprüche, epistemische Zuschreibungen und akademische Praktiken durchzusetzen, um sich von einer rein theoretisch verstehenden und sozialpolitisch enthaltsamen Soziologie abzugrenzen, was aber zur Folge hätte, an gesellschaftlicher Relevanz einzubüßen (Sala 2017, S. $559 \mathrm{ff}$.). Letztendlich ging es aber mit dem Werturteilsstreit darum, ein wissenschaftliches objektives Ideal zu verkörpern, dass es ermöglicht, eine relative Unabhängigkeit von der Politik zu bekommen, was aber nicht bedeutet, auf diesen keinen Einfluss nehmen zu können (Sala 2017, S. 565). Dieses ermöglichte, Soziologie nicht mehr vornehmlich als philosophische Disziplin zu verstehen, sondern sie vielmehr empirisch zu fundieren, was zugleich mit angewandt gleichgesetzt wurde (Sala 2017, S. 567).

6 Diese Frage war auch konstitutiv für die Entstehung der Deutschen Gesellschaft für Soziologie (DGS) 1909 in der Georg Simmel, Max Weber und Ferdinand Tönnies beteiligt waren und welche ein Forum für den Streit einer sich kulturkritisch verstehenden Soziologie und einer tatsachenwissenschaftlichen ausgerichteten Soziologie mit erklärendem Anspruch bildete.

7 So plädierte M. Weber dafür, dass Wissenschaft als ein fachlich betriebener Beruf zu verstehen sei, der ,im Dienst der Selbstbesinnung und der Erkenntnis tatsächlicher Zusammenhänge, und nicht eine Heilsgüter und Offenbarungen spendende Gnadengabe von Sehern und Propheten oder ein Bestandteil des Nachdenkens von Weisen und Philosophen über den Sinn der Welt“" sei (Weber 2018 [1919], S. 33).

8 Das von F. Tönnies (1887) erschienene Buch „Gemeinschaft und Gesellschaft“, das zunächst den Untertitel „Abhandlungen des Communismus und des Sozialismus als empirische Kulturformen“ trug, wurde in der 2. Auflage 1913 umbenannt in „Grundbegriffe der reinen Soziologie“.

9 ,Die Soziologie ist nicht nur eine Wissenschaft mit eigenen gegen alle anderen Wissenschaften arbeitsteilig abgegrenzten Objekten, sondern sie ist eben auch eine Methode der historischen und der Geisteswissenschaften überhaupt geworden“ (Simmel 1920, S. 18).

$10 \mathrm{M}$. Webers Interesse hingegen war eine Soziologie, die als unabhängige Disziplin fungierte. M. Weber konnte sich in den Anfangsjahren der DGS nicht durchsetzen. Das hatte zur Konsequenz, dass er 1911 aus der neu gegründeten Gesellschaft wieder austrat (Lepsius 2011; Sala 2017, S. 566f.).
} 
epistemisches Bezugsfeld die Sozialpolitik gewesen ist. Deren ökonomische und methodisch quantitative Ausrichtung wie auch deren sozialtechnologisch ausgerichteter sozialpolitischer Anwendungsbezug wurde ebenfalls kritisiert (Sala 2017, S. 575). Stattdessen lag das Interesse der führenden Autoren der DGS auf einem philosophisch begründeten gesellschaftswissenschaftlichen, aber auch normativen Zugang (vgl. Sala 2017, S. 576), der Soziologie aus der Staatszentrierung befreien sollte ${ }^{11}$.

Auch in der Psychologie vollzog sich ein ähnlicher Abgrenzungsdiskurs mit der Gründung der Gesellschaft für experimentelle Psychologie, welche aber erst nach dem ersten Weltkrieg zunehmende Bedeutung erfuhr (vgl. Gundlach und Stöver 2004, S. 15). Vertreter der experimentellen Methodik wie Ernst Meumann sprachen der geisteswissenschaftlichen Tradition W. Diltheys aufgrund ihrer Historizität und Subjektivität/Intropsektion ihre wissenschaftliche Legitimität ab (vgl. Wendt 2019, S. 5). Gerade die Frage nach dem Wesen eines Subjekts, die Grundlage der Charakterlogie, wurde als philosophische Frage bestimmt, die aus der psychologischen Gesellschaft möglichst ausgeschlossen werden sollte. Darüber hinaus wurde der experimentellen Psychologie mit ihrer am Labor ausgerichteten positivistischen Methodologie ihr sozialtechnologisch ausgerichteter Anwendungsbezug kritisiert (Heinemann 2016, S. $213 \mathrm{ff}$.).

Der phänomenologische Zugang zur deskriptiven Pädagogik Aloys Fischer kann genauer vor diesen disziplinübergreifenden Entwicklungen, der sich an den Universitäten konstituierenden Sozialwissenschaften, verstanden werden. A. Fischers Interesse bestand - und damit folgte er der phänomenologischen Psychologie Alexander Pfänders - der sich von der Spaltung von beschreibender und erklärender Wissenschaft distanzierte. Begründet wird diese Positionierung damit, dass sich die „Trennung [...] nicht in der ursprünglichen Erfahrung“ (Wendt 2019, S. 4; Fischer 1957b [1925], S. 38 ff.) wiederfände. Das Phänomen der sozialpädagogischen Tatsache wird in der phänomenologischen Tradition weder als innerlich wie bei W. Dilthey, noch als äußerlich wie im Positivismus ${ }^{12}$ betrachtet, sondern vielmehr auf die „Struktur der Erfahrung“ hin befragt.

Von der geisteswissenschaftlichen Tradition grenzte sich A. Fischer ab, da es dieser um eine „Psychologie der wissenschaftlichen Menschenkenntnis“ (Fischer 1957b [1925], S. 37) ging, die den Ausdruck, die Art und Weise wie sich jemand darstellt zum Ausgangspunkt ihrer Forschung nahm. Die methodologischen Regeln wären solche der wissenschaftlichen Deutung, bei der die Historizität aber auch die Subjektivität eine zentrale Bedeutung bekäme (Fischer 1957b [1925], S. 37f.). Von experimenteller Psychologie und der Pädagogik E. Meumanns war A. Fischer zwar angezogen, indem er von der Bedeutung des Experiments für die pädagogische Forschung sprach. Zugleich betonte er aber, in Abgrenzung zu E. Meumann, dass

\footnotetext{
${ }^{11}$ Ich werde zeigen, dass A. Fischer genauso wie G. Simmel die Sozialpädagogik als ,relativ“ eigenständig verstand, womit ein „eklektizistischer“ Zugang zu den Ergebnissen anderer Wissenschaften einherging, welcher die „,verschiedenen Forschungsergebnisse und Theorieteile zu einer neuen Synthese“ (Korte 2004, S. 88) führte.

12 Dieser Tatsachenzugang ,zur Sache“ der Sozialpädagogik selbst, unterscheidet sich von denen der Sozialwissenschaften, wie sich dieses exemplarisch in der Auseinandersetzung mit E. Meumann zeigt (vgl. Fischer 1950 [1914], S. 29), die eben Tatsache, Empirie und Wissenschaft gleichsetzt (vgl. Neumann 2008, S. 185).
} 
die Pädagogik nicht in einer ,exakten' Wissenschaft aufgehen dürfe (vgl. Fischer 1950 [1914], S. 29; Niemeyer 1998, S. 106f.; Neumann 2008, S. 185).

Fischer zielte darauf ab, den Dualismus dieser beiden Wissenschaftstraditionen und den damit eingehenden Subjektivismus und Objektivismus zu überwinden. Dabei ist er aber der geisteswissenschaftlichen Tradition näher als der experimentellen. Anknüpfend an der geisteswissenschaftlichen Tradition der Erkenntnisbildung greift A. Fischer den Bezug auf den Ausdruck auf, richtet aber den Blick von ,,ich drücke mich aus' zu ,es drückt sich (meine Spannung, meine Erregung, meine Art usw.) aus “" (Fischer 1957b [1925], S. 40). Diese Wendung ermöglicht von der Perspektive des Kundgebenden und damit einhergehenden Vorstellung der Introspektion als Methode auf die öffentlich sichtbare Form des Ausdrucks zu wechseln. Dadurch ist der Ausdruck für den „Kundgebenden“ wie den „Auffassenden“ „gleichsinnig“ und „gegensinnig“",real“ (Fischer 1957b [1925], S. 41).

Dennoch stellt sich die Frage, was ,es' ist, das sich ausdrückt. Hier verweist A. Fischer ausgehend von einem biologischen Verständnis des Psychischen auf das Lebendige. ,Seelisches Geschehen ist ein Lebensprozeß, indem alles, was etwas in anderer Betrachtung als Element angesprochen werden könnte, in einer unaufhörlich wechselnden Situationsverschiebung seine Stellung, seine Bedeutung nur aus der wechselnden Gegenseitigkeit alles anderen vorgängigen und gleichzeitigen Geschehens gewinnt“ (Fischer 1957b [1925], S. 43). Diesen Lebensprozess gelte es „kausal zu erklären“ als auch ,teleologisch zu interpretieren“ (Fischer 1957b [1925], S. 44). Es wird deutlich wie A. Fischer hier den phänomenologischen Intentionsbegriff aufgreift, der weniger seinen Ausgangspunkt im Subjekt hat, sondern sich als etwas zeigt, das sich in der ,Schwebe“ hält, erhält und entfaltet (Fischer 1957b [1925]). Dabei spielt es für A. Fischer keine Rolle, ob es hier um die Entfaltung einer Person als Ganzes oder wie in der Soziologie um die Entfaltung eines Gesellschaftsprozesses oder um den Sinnzusammenhang des Erziehungsprozesses geht. All diesen Formen liegt nach A. Fischer letztendlich etwas Lebendiges zu Grunde, welches er organologisch als etwas Ganzes versteht und welches aber zukunftsoffen gestaltet werden kann (Fischer 1954a [1923], S. 30). Der Bezug auf das Lebendige, die organologische Grundlage seiner Erkenntnistheorie macht seine totalitätsbezogene Erkenntnisposition deutlich (Bonß 1983, S. 59), die sich kulturkritisch gegenüber den historisch entstandenen Sozialgestalten zeigt.

Diese Erkenntnisposition zeigt sich auch in seiner Vorstellung von sozialpädagogischer Theoriebildung, die weniger ihren Ausgangspunkt in der Begriffsbildung nimmt, sondern vielmehr geht es ihm um eine theoriebildende Erforschung der sozialpädagogischen Tatsache (Fischer 1950 [1914], S. 7). Dabei ginge es der Tatsachenforschung nicht um eine Verbesserung der Praxis, sondern um die Frage nach der ,Struktur der pädagogischen Praxis“ (Fischer 1950 [1914], S. 7), die aber etwas anderes sei als eine „Anleitung“ oder auch eine ,,Begründung 'von Zielen und Methoden der Pädagogik“ (Fischer 1950 [1914], S. 8). Anstatt eine pädagogische Tatsache als selbstverständlich zu nehmen, müsse erst durch eine philosophisch fundierte Theorie festgestellt werden, welches die Voraussetzung dafür sei, eine Problemstellung wie auch -lösung bilden zu können (Fischer 1950 [1914], S. 14f.). Es wird deutlich, wie A. Fischer strukturanalog zum Diskurs in der Soziologie aber auch in der Psychologie sich von der Vorstellung einer angewandten Wissenschaft abgrenzt 
(Fischer 1957b [1925], S. 34), um Erziehungswissenschaft als relativ unabhängige Disziplin begründen zu können. Zugleich erhebt er den Anspruch praktischer Relevanz, welche bei A. Fischer nur den Bezug auf Theorie ermöglicht.

\subsection{Deskriptive Pädagogik als (sozial-)pädagogisch phänomenologische Erkenntnistheorie und ihre methodologischen Implikationen}

A. Fischers Vorstellungen zur Empirie werden von ihm in der ,deskriptiven Pädagogik' dargelegt. Dort verweist er auf die Bedeutung der phänomenologischen Reduktion, die sich aller „,vorgefasster Absichten und Wertung“, aber auch „Erklärungen“ enthält und vor diesem Hintergrund sich der Struktur der (sozial-)pädagogischen Praxis nähert (vgl. Fischer 1950 [1914], S. 25 ff.). In der erziehungswissenschaftlichen Rezeption A. Fischers wird ihm hier ein regionalontologischer Ansatz zugeschrieben (Lippitz 2020; Brinkmann 2017, S. 20 f.). Die „Struktur der sozialpädagogischen Praxis“ geht von der alltäglichen „Realitätserfahrung“ als „Untergehbares positives Datum“ (Lippitz 2020, S. 157) der Pädagogik aus. Diese müsse ,geglaubt“ werden (Lippitz 2020, S. 157). Damit wird deutlich wie von A. Fischer einerseits aus dem Positivismus die Möglichkeit objektiver Erkenntnis übernommen wird, wenn er von der „Realitätserfahrung“ als „,unterhintergehbares Faktukum“ ausgeht. Aus diesem Grunde verwundert es nicht, wenn er seinen phänomenologischen Zugang in Anlehnung an den Positivismus als tatsachenwissenschaftlich (vgl. Bonß 1983, S. 59) bezeichnet. Andererseits unterscheidet er sich von dem positivistischen tatsachenwissenschaftlichen Zugang, da ein subjektives Moment erhalten bleibt, da das, was als Realität erfahren wird, d.h. die sozialpädagogische Tatsache als reales Phänomen ,geglaubt“ werden soll. Geglaubt werden kann aber nur eine Repräsentation einer Realitätserfahrung und zwar als solche, die dem Gegenstand angemessen ist.

A. Fischer wählt Formen der Beschreibung, die es ermöglichen sollen, dass diejenigen, die diese Beschreibungen lesen, sich von der ,suggestiv wirkenden Beschreibung“ überzeugen können (Fischer 1957 [1914], S. 77 f.). D.h., dass alle, die diese Beschreibung lesen, gegenüber ,gleiche[n] und nach gleichen Richtungen aufgefasste[n] Erlebnisse[n] hinschauen“ (Fischer 1957 [1914], S. 77f.). Die Beschreibung vermittelt somit, so Fischer, nicht nur was etwas ist, sondern auch wie es zu erleben ist. Es gibt die Richtung vor, die intersubjektiv in gleicher Weise erfahren wird und es dadurch ermögliche, die Wahrheit der Erziehung selbst zu erschließen (vgl. Fischer 1957b [1925], S. 41; Lippitz 2020, S. 163). Die Möglichkeit, dass verschiedene Personen durch die Beschreibung das gleiche erfahren, ist bei A. Fischer somit die Bedingung der Möglichkeit, sich als Tatsachenwissenschaft begründen zu können. Zugleich bedeutet es aber auch, dass es sich hier um eine totalitätsbezogene Erkenntnisproduktion handelt. Sie basiert auf der vorgängigen Erfahrung der vergesellschafteten Sozialpädagogik als Tat, bei der, wie ich später zeigen werde, moralische Gefühle, Willen, von zentraler Bedeutung sind und die es wissenschaftlich zu erforschen gilt (vgl. Bonß 1983, S. 58f.). In der deskriptiven Pädagogik geht es letztendlich darum, eine Einsicht über die sozialpädagogische Tatsache im Bewusstsein zu erzeugen. Bewusstsein bedeutet aber ein ,kreativer gegen die Zukunft hin offener" (Herzog 1992, S. 131f.) Erkenntnisakt. Damit geht einher, dass trotz des Anspruchs auf Objektivität, diese nicht naturwissenschaft- 
lich begriffen wird, sondern es hier vielmehr um eine theoretisch vermittelte wie empirisch grundgelegte Herstellung von Sozialpädagogik als Sinnzusammenhang geht. Geprüft werden somit empirische Erkenntnisse der Sozialpädagogik darauf, welchen Beitrag sie zur Einsicht in den sozialpädagogischen Sinnzusammenhang leisten. Da A. Fischer sich von der Dualität von geisteswissenschaftlicher Pädagogik wie tatsachenwissenschaftlicher empirischer Pädagogik verabschieden möchte, zeigt er sich offen gegenüber den verschiedenen methodologischen Zugängen zur Empirie der Pädagogik (Herzog 1992, S. 28). Diese Offenheit ist aber nicht gänzlich entgrenzt, sondern rückgebunden an die Adäquatheit der Deskription der Erfahrung der Struktur der Sozialpädagogik (Wendt 2019, S. 5).

Dann stellt sich aber die Frage wie sich die gegenstandsbezogene Theorie der Sozialpädagogik (Abschn. 2.3), welche einerseits vorausgesetzt wird, andererseits aber in der phänomenologischen Reduktion eingeklammert wird, zur empirischen Erkenntnisproduktion verhält. Einerseits braucht es eine Offenheit für die empirischen Studien, die das, was schon von Sozialpädagogik gewusst wurde, überschreitet, andererseits muss sich das, was den Anspruch hat, einen forschenden Beitrag zur Sozialpädagogik zu leisten, sich in den Horizont sozialpädagogischer Erfahrungsbildung einreihen.

Das Verhältnis zwischen Tatsächlichkeitsbezug und Reflexivität ${ }^{13}$ gestaltet sich bei A. Fischer in folgender Weise. Das Reale gerät mit dem Glauben oder dem fiktiven Charakter der Deskription der Sozialpädagogik in ein Spannungsverhältnis. Der fiktive Charakter, d.h. die zu generierende Überzeugung, verweist auf die Hergestelltheit der Sozialpädagogik als Sinnzusammenhang. Die Hergestelltheit wird umso deutlicher, weil A. Fischer die (sozial-)pädagogische Tatsachenforschung nicht durch Methoden im engeren Sinne absichert. Gerade deswegen hat er die Beschreibung der Sozialpädagogik zu verantworten. Damit rückt der Blick von Was Sozialpädagogik ist auf das Wie wird Sozialpädagogik im Bewusstsein zwischen Autor und Publikum hergestellt. Es handelt sich um eine Form „dichter Beschreibung“ (Geertz 1987), eine ,interpretative Rekonstruktion“ (Wolff 1992, S. 344) der affektiven Erfahrungen und Bedeutungsstrukturen der darin involvierten Akteur*innen, die als „Schaustücke“ der sozialpädagogischen Tat zur Darstellung gebracht werden und eine ,evozierende“ Funktion haben (Wolff 1992). A. Fischer ist sich dieser Spannung zwischen einer ,interessenslosen“ Beschreibung (Fischer 1950 [1914], S. 7), um ,zu den Sachen“ selbst zu kommen und der perspektivischen Beschreibung (Lippitz 2020, S. 164) durchaus bewusst, wenn er darauf verweist, dass sich die

\footnotetext{
13 Einerseits scheint es A. Fischer um die Realität der Erfahrung zu gehen, andererseits um einen Glauben in das, was sich zeigt, welches es so zu beschreiben gilt, dass es von anderen in gleicherweise nachvollzogen werden kann und eine Einsicht in Wie sich Sozialpädagogik vollzieht generiert.
} 
Wahrheit des Gegenstandes nicht unmittelbar, sondern nur vermittelt erschließt, da sie in weitgehend unbewusster ${ }^{14}$ Form vorliege (Lippitz 2020, S. 163 f.).

\section{Zur Struktur der sozialpädagogischen Erfahrung Aloys Fischers}

Die Struktur der sozialpädagogischen Praxis vollzieht sich als Tatsachenzusammenhang in der gesellschaftlich-geschichtlichen Welt. Sie wird in ihrer Wirkung erlebt. A. Fischer erfuhr die Struktur der sozialpädagogischen Praxis biographisch. Für ihn war die Schule ein pädagogisches Milieu, welches ihm ermöglichte, vom Milieu des Tagelöhners in das des Adels als Prinzenlehrer zu wechseln, ohne dadurch zum Adel zu werden (Kreitmair 1954). Diese Erfahrung, d.h. die Tatsche des sozialen Erlebens von Differenz und Zusammengehörigkeit bildet das implizite Wissen über Sozialpädagogik, welches ihm ,realistisch“ erscheint, da er dieses selbst erlebt hat. Durch die Auseinandersetzung mit den Sozialwissenschaften versuchte Fischer sein implizites Wissen zu explizieren ${ }^{15}$.

A. Fischers Aussage, dass das soziale Milieu nicht determiniere, sondern die Milieupädagogik der Schule eine Wirkung entfaltet, wird angesichts seiner Erfahrungen des sozialen Aufstiegs plausibel. Von hier aus bestimmt er die Bedeutung der pädagogischen Soziologie, welche analysiert, wie sich das (sozial-)pädagogisch gestaltete Milieu von anderen Formen gesellschaftlicher Sozialisation unterscheidet (vgl. Fischer 1957a [1915], S. 101). Die Möglichkeit des Aufstiegs verortet A. Fischer aber nicht in der didaktischen Gestaltung des Unterrichts und damit der Individualpädagogik. Vielmehr sei es notwendig, den Blick über die Individualpädagogik hinaus, die die Erziehung als intentionalen Akt versteht, zu weiten, indem die Aufmerksamkeit auf den Erziehungsstil eines Milieus gerichtet wird, und von dem eine erzieherische Wirkung ausgehe. Genau diese Abgrenzung ist die theoretische (teil)disziplinäre Begründungsfigur der Sozialpädagogik zu Beginn des 20. Jahrhunderts gewesen (Reyer 2002). Eine Soziologie der Erziehung nach A. Fischer analysiere und klassifiziere empirisch die für die Erziehungsmöglichkeiten angemessenen Sozialverhältnisse (vgl. Röhrs 1967, S. 248).

Diese Formungskräfte bezeichnet A. Fischer als Milieus. Da von Milieus eine Erziehungswirkung ausgehe, gelte es, diese so zu gestalten, dass schädigende Wir-

\footnotetext{
14 Das Unbewusste wird bei A. Fischer in dreifacher Form konkretisiert: erstens sei die Beschreibung durch die gesellschaftlichen bzw. milieuspezifischen Verhältnisse vermittelt, die dieses prägen, aber nicht determinieren, da diese ,durch prälogisch-assoziative, fantasieförmige, bildhafte Verkettungen weitergebildet“ (Lippitz 2020, S. 163) werden. Zweitens seien Beschreibungen durch das Unbewusste im Sinne der Psychoanalyse geprägt und drittens durch das Unbemerkte im Sinne, dass das Einzelne angesichts der Gestaltschließung übersehen würde (Lippitz 2020). Durch diese Verweise auf die kreativen Momente, wird deutlich wie von A. Fischer die Beschreibung der Sozialpädagogik dekonstruktiv realisiert wird, weil sie das, was als Sozialpädagogik bestimmt ist, überschreitet. Gerade darin zeigt sich A. Fischers Verantwortung als Autor, da er im Unterschied zur naturwissenschaftlich geprägten Darstellungsweise sich zwar als beschreibend, aber in der Reflexivität als zukunftsweisend begreift und dazu braucht es eine gestaltende Darstellung der Sozialpädagogik, die die Sozialpädagogik wie sie intentional sein will (re-)präsentiert.

15 J. Reyer verweist, darauf, dass nicht nur A. Fischer, sondern viele Lehrer*innen zu dieser Zeit ,Gelegenheitssoziologie' betrieben haben, da ihre Erfahrungen mit der pädagogischen Arbeit das Interesse an den sozialen Wirkungskräften formte (vgl. Reyer 2002, S. 165 f.).
} 
kungen durch erzieherische ersetzt werden würden. Dabei käme es darauf an, dass den „Heranwachsenden ein Verständnis der Gemeinschaften, zur sozialen Dienstgesinnung, zur Solidarität mit den Menschen seiner verschiedenen Sozialkreise“ durch den ,Einbau von Gelegenheiten“, also vermittelt und nicht unmittelbar erzogen würde. Dabei stellt sich aber nach A. Fischer eine Soziologie der Erziehung zur Frage, wie die Möglichkeiten der Sozialpädagogik im Verhältnis zum gesellschaftlichen Milieu zu beurteilen wären. Ziel sei es, die „Muster [...] unbewußt erzieherisch wirkender Sozialkreise“, die die „Kunstformen der bewußten Erziehung selbst“ prägen, empirisch zu rekonstruieren und reflexiv, d.h. unter Berücksichtigung der gesellschaftlichen Bedingungen, zu gestalten (Fischer 1954d [1932], S. 166).

A. Fischer strebt eine Bewusstseinsbildung von einer unbewussten, selbstverständlichen gesellschaftlichen Stellung einer Person in einer Gesellschaft bzw. in einem (pädagogischen) Milieu zu einer bewussten, sich dazu ins Verhältnis setzenden Person, angesichts der sozialen Erlebnisse in einem (pädagogischen) Milieu an. Das soziale Erleben kann, so A. Fischer, durch die Sozialpsychologie ${ }^{16}$ als Teil der soziologischen Pädagogik empirisch erschlossen werden und durch die ,Psychoanalyse ${ }^{\prime} \mathrm{zu}$ einem Bildungsakt werden. A. Fischer spricht im Zusammenhang der Sozialpädagogik von einem sozialen Bewusstsein, welches ein Bewusstsein von Gesellschaft sei, welches als teils ,naturhaft“ teils als ,geschichtliche Wirklichkeit“" (Fischer 1954b [1924], S. 257) gegeben ist. Bezugnehmend auf das Unbewusste führt A. Fischer aus: „Nicht jeder muß bewußt und unmittelbar dem Ganzen dienen und dienen wollen, aber wer ohne soziales Bewußtsein lebt und handelt oder trotz desselben sich so benimmt, als ob er Einziger auf der Erde wäre [...] lebt mit dem schlechten Gewissen verdrängter besserer Einsicht oder der primitiven Lüge des Bewußtseins, die alles ,Vorgestellte ' nur für ,Verstellung ' hält" (Fischer 1954b [1924], S. 258). Auf der einen Seite erscheint es so, als ob Fischer ein soziales Bewusstsein ontologisch setzt, auf der anderen Seite verweist er darauf, dass dieses Bewusstsein zu vermitteln sei und dieses die Aufgabe der verberuflichten (Sozial-)Pädagogik sei.

Die Schule wird für Fischer der zentrale Ort, indem das bisherige naive soziale Bewusstsein zu einem reflexiven werden kann ${ }^{17}$. Dieses sei dann nicht mehr „gesellschaftsauffassend“, sondern ,gesellschaftsgestaltend“ (Fischer 1954b [1924], S. 258). Dazu braucht es aber die Schule als Institution gegenüber der Gesellschaft und ihren ,Organen“, welche ein gestaltendes Bewusstsein ermögliche (vgl. Kreitmair 1954, S. 20). Hier entwickelt A. Fischer für Sozialpädagogik einen Ansatz, der auch mit Lawrence Kohlberg (1984) als ,Just-Community-Ansatz beschrieben werden könnte, der sich zwar auf seine Schulerfahrung bezieht, zugleich aber über diese hinausgeht. Voraussetzung für die Bildung eines sozialen Bewusstseins ist, dass in

\footnotetext{
${ }^{16}$ Für A. Fischer wurde die Sozialpsychologie von zentraler Relevanz für die Sozialpädagogik, da sie auf die Bedeutung des Milieus für die Erziehung verweist. Der Sozialpsychologie als „Psychologie der Gesellschaft" geht es darum, wie das gesellschaftliche Milieu, die Klassenlage oder das pädagogische Milieu in der Schule etc. erlebt wird.

17 Die gouvernementale Form ,ganzheitlicher ${ }^{\star}$ Bildung für die Gesellschaft durch Schule unterscheidet sich von der Johann Heinrich Pestalozzis, da dieser mit seiner ständischen und familiären Erziehung das Transformationspotenzial der Schule nicht entfalten kann (vgl. Fischer 1954b [1924], S. 224). Zugleich unterscheidet sich A. Fischer aber auch von Herman Nohl, der im ,pädagogischen Bezug“ letztendlich auch die Familiarität als Orientierungsmuster für die Sozialpädagogik nimmt (Lütke-Harmann 2016, S. 180 ff.).
} 
der Schule nicht nur ein intellektuelles Verständnis von dem sozialen Bewusstsein vermittelt wird, hier distanziert er sich von der gymnasialen Bildung, sondern es um die Bildung von Willen und Werten zu einer demokratischen Gesellschaft in der Schule gehe ${ }^{18}$. Die Schule müsse dazu als eine ,,selbstverwaltende Rechtsgesellschaft“ organisiert werden, die durch „Selbstgesetzgebung, Selbstverwaltung und Selbstjustiz“ (Fischer 1954b [1924], S. 237) funktioniere. Sie müsse als demokratische Ordnung institutionalisiert werden, was auch möglich sei, bevor sie sich als Staatsverfassung etabliert habe. A. Fischer schwebt hier eine Form gouvernementaler Regierung vor, eine Einsicht in die soziale Ordnung ohne Zwang (vgl. Fischer 1954b [1924], S. 237). Schule sei somit eine ,praktische Rechtsschule“, die eine „Vorbildung“ für die staatsbürgerliche Welt sei, da durch eine solche Erziehung die Menschen ,empfänglich“ für eine „,konstitutionelle Verfassung“ seien (Fischer 1954b [1924], S. 238f.). Damit bestimmt A. Fischer die organisatorischen Voraussetzungen für die Bildung des sozialen Bewusstseins. Zugleich verweist er aber darauf, dass diese alleine nicht ausreichen, sondern eine ,Sittenklasse als moralische Hilfsanstalt" ebenfalls notwendig sei. Es gelte, die Schüler*innen auf ihren sittlichen Fortschritt hin zu bewerten, was durch „Sittenräte“ (Fischer 1954b [1924]), die von den Schüler*innen selbst gebildet werden, vollzogen werden könnte. Dadurch würde nicht nur die Vernunft, sondern auch die „Seele“ und der „Charakter“ erzogen. Je mehr Schule demokratisiert sei, desto mehr würde dieser Stil auch als Milieu auf Persönlichkeit übergreifen, welche diese Ideale mit Überzeugung trage (vgl. Fischer 1954b [1924], S. 241). Es wird deutlich, wie die Normierung durch die Sittenräte als Sachzwänge für das Leben in einer Gemeinschaft dargestellt werden und somit die Normen selbst nur bedingt in der Form des Als-ob diskursiv verfügbar sind, da sie ontologisch gesetzt zu sein scheinen. Zugleich wird aber auch deutlich, dass es hier um Praktiken geht, die das soziale Bewusstsein ermöglichen sollen und dass die ,gelingende" Form empirisch zu rekonstruieren sei, und damit von A. Fischer nicht schon immer gewusst sein kann. Vielmehr geht es ihm im Anschluss an G. Simmel um einen offenen historisch situierten Prozess. Dadurch wäre aber ein reflexiver Blick im Hinblick auf die Folgen, die von solch gouvernementaler Regierung ausgehen (Flösser und Wohlgemuth 2011, S. 1370f.) denkbar, sofern die Folgen durch die pädagogische Soziologie zuvor empirisch sichtbar gemacht werden würde.

Die gouvernemental gestaltete Schule bekommt für A. Fischer eine zentrale Bedeutung für die Überwindung einer ,ständisch ' geprägten sozialen Ungleichheit in der Gesellschaft, welche er angesichts der davon ausgehenden Konfliktpotenziale immer wieder kritisiert. Aus diesem Grunde wurde von A. Fischer diese Gleichheit ermöglichende Gestaltungsaufgabe einer verberuflichten Sozialpädagogik zugeschrieben, die letztendlich zur Erneuerung der Schulpädagogik beitragen sollte, damit dieser ihrer Erziehungsfunktion gerecht werden könne (Niemeyer 1998, S. 114). Dabei verfolgte er das Ziel, dass die Ausdifferenzierung der Pädagogik in Schulpädagogik und solche der Jugendfürsorge und Jugendpflege, wie von Marie Bäumer vertreten, sich im Sinne einer ihrer Erziehungsfunktion gerecht werdenden

18 Im Anschluss an die im Vormärz erschienen Veröffentlichungen zum „System der öffentlichen Erziehung“ des evangelischen Pfarrers und Schulreformers Heinrich Stephanis (1805), spricht A. Fischer von staatsbürgerlicher Erziehung. 
,ganzen“ Pädagogik wieder entdifferenzieren sollte (Fischer 1967a [1930], S. 464). Diese verstand er präventiv und verband sie mit der Vorstellung, dass durch Erziehung soziale Abweichungen verhindert werden könnten, und damit eine soziale Fürsorge überflüssig wäre (Fischer 1967a [1930], S. 463).

\section{Zur (sozial-)pädagogischen Verberuflichung}

Unter Beruf versteht A. Fischer nicht einfach Erwerbsarbeit, sondern vielmehr Berufung: „Der Beruf im tiefsten, sittlichen Verstand ist die Erfassung einer lebenslänglichen, innerlich verbundenen Tätigkeit, durch welche der einzelne sich selbst im Werk restlos ausformt“", die aber zugleich einem größeren Sozialsystem wie dem „wohlfahrtstaatlichen System [aber auch dem der Wissenschaft; B. H.] diese Kräfte zur Verfügung stellt, so dass dieses sich entwickeln kann“ (Fischer 1954b [1924], S. 245). Der Beruf, der Bezüge zur Sozialpädagogik aufweist, ist der des Sozialbeamten, der in Deutschland die Fürsorgearbeitet leistet. Diesem Berufsstand steht A. Fischer skeptisch gegenüber, da durch Fürsorgearbeit die Gefahr bestehe, dass diese zu bürokratisch sei (vgl. Fischer 1954c [1925], S. 328). Er kritisiert, dass Fürsorge kein freier Beruf sei, der es ermöglicht, dass die Hilfebedürftigen, sich ihre*n Fürsorger*in aussuchen, sondern, dass sie sich mit der Person arrangieren müssen, die ihnen zugeordnet wird (vgl. Fischer 1954c [1925], S. 322). Dies widerspräche der zentralen Voraussetzung für das Gelingen der Fürsorgearbeit, dem Vertrauensverhältnis zwischen Fürsorgeempfänger*in und Fürsorger*in (vgl. Fischer 1954c [1925], S. 323). A. Fischer problematisiert weiterhin am Sozialbeamtentum, dass diese zu sehr an Einzelfällen orientiert seien, anstatt auf das „Ganze der Lebenskreise“ (Fischer 1954c [1925], S. 334) einzuwirken. Dieses sieht er am ehesten in der Settlementbewegung oder in Deutschland in der Nachbarschaftsbewegung verwirklicht (Fischer 1954c [1925]).

A. Fischer versteht Sozialpädagogik als ein Experiment: „Die Versuchsmäßigkeit, die Schwebe zwischen einem dauernd vorhandenen, ein[em] naturhaften Ausgangspunkt und einem in immer höhere Formen rückenden, ausschließlich geistbestimmten Ziel, schließt mit Notwendigkeit nicht nur den möglichen zivilisatorischen, kulturellen und ethischen Fortschritt ein, sondern ebenso auch das unvermeidliche Versagen und Absinken von einzelnen oder Gruppen von solchen" (Fischer 1954c [1925], S. 344). Deutlich wird, dass A. Fischer weder klare Maßstäbe hat, nach dem das Gelingen beurteilt wurde, noch die Vorstellung, dieses systematisch hervorbringen zu können, sondern, dass vielmehr ein Lernen aus den Erfahrungen notwendig wäre. Allerdings sieht er durchaus soziale Bedingungen, unter denen das Experimentieren leichter wäre und verweist darauf, dass dieses eher schwierig sei, wenn es besonders ,schwere ' Fürsorgefälle wären. Aus diesem Grunde hält er eine präventive Orientierung für umso notwendiger (vgl. Fischer 1954c [1925], S. 337).

Es stellt sich aber die Frage wie ein solcher Berufsethos ermöglicht, d.h. ausgebildet werden kann. Voraussetzung dafür ist ein Anknüpfen an die eigene Biografie, die aber zugleich im Spannungsverhältnis zum Berufsethos des Berufsbeamten steht. „Aber es leuchtet ein, daß die außerberufliche Artung wesentliche Erleichterungen bzw. Erschwerungen für die Entstehung und Bildung der Berufsseele, für die täg- 
liche Berufsübung mit sich bringt, die in der Ausbildung für den Beruf entweder abgeglichen oder wenigstens für die berufliche Selbsterziehung sichtbar gemacht werden müssen" (Fischer 1957d [1926], S. 320).

Letztendlich thematisiert A. Fischer an dieser Stelle die unterschiedlichen Sozialisationswirkungen durch das Aufwachsen einer Person in Relation zur tertiären Bildung. Letztere sieht er aber nicht entlastet von der Erziehungsaufgabe, sondern vielmehr plädiert er dafür, dass auch diese von der Erziehungsaufgabe durchdrungen wird (vgl. Fischer 1967b [1932]). Als tertiäre Bildung liegt der Schwerpunkt auf der höheren Sinn(es-)bildung, d.h. der Ermöglichung eines Berufsethos, welcher die biografischen Sozialisationserfahrungen reflexiv durchdringt. Letztere werden aber nicht nur als Problem, sondern auch als Möglichkeit betrachtet, sofern diese „Mittel und Werkzeug" beruflichen Handelns würden (Fischer 1957d [1926], S. 323 ff.). Von solchen Formen der habituellen Repräsentation, A. Fischer spricht hier von „Vorbild“, gehen sozialisierende Wirkungen aus, unabhängig von der Intention, die dem Handeln zugrunde liegt.

Zur Herausbildung des Berufsethos braucht es eine Akademisierung, ,weil der ängstliche Zuschnitt der Ausbildung auf das sofort Anwendbare und das unmittelbar Berufsbrauchbare eben jene Enge des Menschentums nach sich zieht, die den pädagogischen Handwerker auch noch in seiner fachgerechten Musterform von der Berufsidee des Erzieher- und Lehrertums notwendig abkehrt" (Fischer 1957d [1926], S. 314f.). Grundlage sei ein „Berufethos“, der der „Berufstechnik“ übergeordnet sei (Fischer 1957d [1926], S. 316) und nur durch eine höhere Bildung, geschult" werden könne. A. Fischer unterscheidet hier zwischen einem akademischen Habitus, der durch eine universitäre Bildung ermöglicht würde und der sozialen Wirklichkeit der (sozial-)pädagogischen Arbeit. Zwischen diesen Realitätsebenen muss, so A. Fischer, letztendlich der pädagogische Ruf, das Ge-Wissen vermitteln. Dabei wird die Vermittlungsarbeit als gelernte und habitualisierte Kunst der Bildungsarbeit bezeichnet (vgl. Fischer 1957d [1926], S. 319), wobei derjenige, der dieses tut als „Professionist“ (Fischer 1957d [1926], S. 320) bezeichnet wird. Dieser würde nicht nur den Blick auf die pädagogische Arbeit selbst richten ,für die Zielung und Verzahnung, den Ausgleich und die Abstimmung auch aller anderen Beiträge zum Bildungsprozess, für die Erziehung als eine ihrer Idee nach einheitliche sich verantwortlich zu fühlen" (Fischer 1957d [1926], S. 320). Es wird deutlich, wie im Berufsethos eine über das pädagogische Geschehen hinaus, sich auf das pädagogische Milieu beziehende Verantwortung übernommen werden soll. Dies wird von A. Fischer als spannungsreich wahrgenommen, was nicht zuletzt daran liegt, dass der Berufsethos auf das Ganze der sozialen Wirklichkeit zielt, für die man als vermittelnde Führungskraft zwischen sozialpädagogischer Innenwelt (pädagogisches Milieu als Ge-Wissensfrage) und gesellschaftlicher Wirklichkeit verantwortlich ist. Die höhere Sinnbildung des Berufsethos vollzieht sich nicht durch Anleitung, vielmehr geht es um eine Vermittlung zwischen sozialpädagogischem Wissen und der sozialpädagogischen Tatsache. 


\section{Zur sinnlichen Erkenntnisbildung der Sozialpädagogik}

A. Fischer wechselt von einer gegenstandsbezogenen Theorie der Sozialpädagogik, die er einzuklammern versucht, indem er von den ,,vorgebrachten Theorien“ (Fischer 1950 [1914], S. 12) absieht und schaltet auf die Form des Sehens, des Hörens um, d.h. der sinnlichen Wahrnehmung, welche ihm die, wahre 'Erkenntnis des, Wesens' der (Sozial-)Pädagogik (Fischer 1950 [1914]) zu vermitteln vermag (Aisthesis). Wenn er von der ,phänomenologischen Durchbildung“ (Fischer 1950 [1914], S. 14) spricht, meint er genau diese "Schule des Sehens“ und „Hörens“ als Erkenntnisprozess, um ,die Tatsachen“ des Gebietes der (Sozial-)Pädagogik „festzustellen“19 (vgl. Fischer 1950 [1914], S. 13f.). Zwar kann, wie W. Lippitz es in der Auseinandersetzung mit A. Fischer anbringt, problematisiert werden, dass die Fundierung der wissenschaftlichen Pädagogik sich aus einem „materialen Apriori“ (Lippitz 2020, S. 168) den Organen der sinnlichen Wahrnehmung ergibt, interessant erscheint aber an dieser Konzeption, dass A. Fischer mit der sinnlichen Erkenntnisbildung von der Frage „Was ist (Sozial-)Pädagogik“ auf „Wie ist (Sozial-) Pädagogik“ umstellt. Der Blick richtet sich somit auf das Wie der Geordnetheit und Wirkung der vergegenständlichten Sozialpädagogik im sinnlichen Erleben. Damit verweist A. Fischer auf die leiblich habituelle Seite der Erkenntnisbildung, die er in Relation zum Sinnganzen stellt. Im Fokus steht, wie die vergegenständlichte Sozialpädagogik als Geordnete repräsentiert wird. A. Fischer stellt sich einerseits die Repräsentation als Form der Abbildung im Bewusstseinserleben als eine Übereinstimmung von Repräsentation der Sache im Erleben und der Sache selbst vor. Allerdings verweist er darauf, dass in der Repräsentation insofern eine Verschiebung zur Präsentation stattfindet, da etwas „als“ etwas erscheint (Fischer 1950 [1914], S. 21), es sich erst dadurch als „Innerliches festhaltend [...] zu qualifizieren“ vermag (Fischer 1950 [1914]). Da aber diese „Aufgabe niemals voll erfüllbar“ ist, kann es „,nicht eigentlich beschrieben sondern nur aufgezeigt werden“ (Fischer 1950 [1914], S. 21). Dadurch geht A. Fischer letztendlich über die „Wesensschau“ hinaus. A. Fischer begründet das Aufzeigen als wissenschaftliche Erkenntnisform der (Sozial-)Pädagogik damit, dass letztendlich aufgrund der Unmöglichkeit der deskriptiven Methoden „zu den Sachen" selbst zu kommen, dieser eine Differenz zwischen Sein und Aufzeigen zugrunde liegt. Damit bedeutet bei ihm sozialpädagogische Theoriebildung nicht Sozialpädagogik auf den Begriff zu bringen, da dieses nur dem Selbstzweck der

\footnotetext{
19 A. Fischer beschreibt das Verhältnis zwischen Individuum und Gemeinschaft strukturanalog zur Musik, um den Sinnzusammenhang genauer bestimmen zu können: „Wir entdecken, daß die Zwecke, die der auch isoliert denkbare Mensch verfolgt, allein durch die Tatsache, dass er erlebend und handelnd auf zunächst ebenso isoliert denkbare Menschen mit gleichen Zwecken trifft, zu kollektiven Zwecken werden. [...] Wir sehen also wie ein Stück Gesellschaft aus dem Erlebnis heraus entsteht, nämlich der Zweck als motivische Wurzel eines flüchtigeren oder dauernden Sichverbindens von Individuen, ein gemeinsamer Wille desselben Zweckes an die Stelle der zahlreichen Einzelwillen desselben“ (Fischer 1954b [1924], S. 260). Dabei stellt sich „Verbundenheit mit anderen als mögliche Form des eigenen Lebens ein“ (Fischer 1954b [1924], S. $260 \mathrm{ff}$.), d. h. kultursoziologisch betrachtet als Lebensform, die durch eine performative Kultur dem Milieu als Sinnganzen gestiftet wird. Sie ermöglicht einer ,deskriptiven“ (Sozial-)Pädagogik erst von einer gegenstandsbezogenen Beschreibung zu der eines Formalobjekts überzugehen, die die Gegenständlichkeit, d.h. die Wirkung in Raum und Zeit in den Blick nimmt. Diese bildet nach A. Fischer die eigentliche Grundlage einer wissenschaftlichen Pädagogik.
} 
Theoriebildung diene, sondern vielmehr sozialpädagogische Theorie durch dichte Beschreibung zu generieren.

Wie man zur (sozial-)pädagogischen Erkenntnisbildung kommt, macht A. Fischer in der ,deskriptiven Pädagogik ' an einem Fallbeispiel deutlich. Damit konstituiert er die Falldarstellung und -analyse als Erkenntnismethode, welche einem ermöglicht, von einem einfachen Sehen von etwas (Sozial-)Pädagogischen zu einem höheren Sehen, der Geordnetheit der vergegenständlichten Sozialpädagogik, zu kommen. Der Blick richtet sich auf das Zeigen, wie das soziale Geschehen erzieherisch wirkt und in welchem Verhältnis dieses zur Struktur der Sozialpädagogik steht. Ich werde im Folgenden dieses Fallbeispiel als (Sozial-)Pädagogisches aufgreifen, d.h. es auf die Struktur der Sozialpädagogik hin verdichten. Dabei werden aber die Widersprüche zwischen dem Fallbeispiel und seiner theoretischen Grundlegung der Sozialpädagogik deutlich. Da es ihm aber um eine Methodologie sozialpädagogischer Erkenntnisgenerierung geht, die sich von einem positivistischen Zugang unterscheidet, ist die Abweichung von seinem sozialpädagogisch-sozialwissenschaftlich geschulten Blick nicht nur ein Problem, sondern eine Möglichkeit für die Re-Vision seiner Vorstellung von Sozialpädagogik.

A. Fischer zeigt in dem von ihm gewählten Fallbeispiel, wie einerseits der Sohn ein Abbild seines Vaters ist und wie andererseits dieses Augenscheinlich-Werden der Spiegelung des Vaters, in einem dem Vaterselbst nicht sympathischen Zug im Sohn, zur „Besinnung“ (Fischer 1950 [1914], S. 23) des Vaters beiträgt, die sich dann in einer „ohrfeigenden“ Geste des Vaters gegenüber dem Sohn zeigt. D.h., das gleiche Verhalten, das er an sich selbst kennt, ahndet er gegenüber dem Sohn. An diesem Beispiel sucht er (sozial-)pädagogische Tatsachenforschung aufzuzeigen, die einen Einblick in die Struktur der (Sozial-)Pädagogik gibt. A. Fischer zeigt auf, dass sich (soziale) Erziehung vollziehe, auch wenn sie nicht absichtsvoll sei, solange sie eine Wirkung zeige (Fischer 1950 [1914], S. 25). Die unbewusste Geste - die Ohrfeige - verweist auf eine Differenz zwischen sich und dem anderen, dass er dem Sohn nicht erlaubt in gleicherweise unsympathisch zu sein, wie er sich selbst als unsympathisch erlebt. Damit ist der Akt des Verbots eines, das sich nicht nur an den Sohn richtet, sondern ihm zugleich sein unmögliches (unsympathisches und damit nicht weiter fortzusetzendes) Handeln durch die Ohrfeige widerspiegelt. Damit gilt das was sich als Orientierungswert in der Ohrfeige indexikalisch zeigt, welche sich auf das Unterlassen einer, unsympathischen“ Handlung bezieht, trotz der generationalen Differenz für beide in gleicher Weise. Der Geste liegt eine Sollensrichtung, eine Indexikalität ${ }^{20}$ zu Grunde. Der Konsens zwischen Vater und Sohn liegt in der Sache eines nicht gewollten Verhaltens, welches nicht verhandelt, sondern durch die ohrfeigende Geste erwirkt wird. Damit zeigt sich die Erziehung nicht nur als Repräsentation von Normen und Werten (Vorbildfunktion), sondern auch als (Re-)Präsentation dieser (Milieubildung). Gerade durch diese Form der Vergegen-

\footnotetext{
${ }^{20}$ Harold Garfinkel versteht unter der Indexikalität in Anlehnung an Edmund Husserl „Gelegenheitsausdrücke“ (Kruse 2009), um die situative Vagheit eines jeden Begriffs und vor allem einer Geste zum Ausdruck zu bringen. Innerhalb der situativ-kontextuellen Dimension von Indexikalität zeigt sich, dass die Bedeutung eines Begriffes und einer Geste nur in seinem konkreten Verwendungskontext erschlossen werden kann.
} 
wärtigung entfaltet sich eine sozialisierende Wirkung auf Vater und Sohn, welche sich paradoxerweise gerade in der erzieherischen Gewalt des generationalen Verhältnisses, in A. Fischers Interpretation eine neue Möglichkeit der Verbundenheit, eröffnet.

Dabei kommt es A. Fischer nicht darauf an, dass die ,Ohrfeige“ ein als schlecht zu bewertendes pädagogisches Mittel sei, wie das gegenwärtig als Kinderrechtsverletzung betrachtet werden würde. Eine solche historisch zu konkretisierende Bedeutung geht A. Fischers ,deskriptiver Pädagogik' nicht voraus, sondern folgt ihr vielmehr (vgl. Fischer 1950 [1914], S. 20), ist also gegenüber der Sache der Sozialpädagogik von sekundärer Bedeutung, sofern das Kind diese Geste als Möglichkeit für sich und nicht als absichtsvolle Strafe (vgl. Fischer 1950 [1914], S. 26) erlebt.

Es wird von A. Fischer ein höherer Sinn aufgezeigt, der die scheinbare Ungeordnetheit des Handelns für beide in eine neue Ordnung überführt, an die sich Vater und Sohn in ihrer Selbstregierung zukünftig freiwillig zu halten haben (Sittengesetz), um ein strafendes Handeln als absichtsvolle Erziehung (durch den ,fiktiven“ von beiden einberufen werden könnenden Sittenrat) zu verhindern. Dadurch wird eine ,just-community“ ermöglicht, d.h. eine Verbundenheit zwischen Vater und Sohn, die durch das (sozial-)pädagogische Milieu hergestellt wird. Von diesem können für beide erzieherische Effekte hinsichtlich sittlichen Handelns ausgehen, wenn auch auf unterschiedliche Weise. Während es für den Vater nicht nur das Unterlassen des unsympathischen Verhaltens, sondern darüber hinaus auch die Unterlassung der strafenden Erziehung bedeutet, ermöglicht es für den Sohn eine Distanzierung von der Mimikry zum Vater, indem er sich bewusst zu dem, was er beim Vater sieht, ins Verhältnis setzt. Dadurch zeigt sich, wie Individuierung und Vergemeinschaftung zugleich möglich sind.

Mit dem Fallbeispiel kann über A. Fischer hinausgehend zwar aufgezeigt werden, wie es gelingt von einem Fall zu einer (sozial-)pädagogischen Tatsachenforschung zu kommen, die zugleich Grundlage dafür wird, Gegebenes in Aufgegebenes zu transformieren - aber mit diesem Fall legitimiert Fischer, dass der Zweck - die Gleichgesinntheit in der Familienerziehung, in dem Fallbeispiel dargestellt als Wirkung - den Zweck der Sozialpädagogik legitimiert. Damit würde der Zweck das Mittel der Ohrfeige ,heiligen“ und somit rechtfertigen. Die ,deskriptive“ (Sozial)Pädagogik wird so zu einer rechtsetzenden Gewalt die angesichts von A. Fischers gegenstandsontologischen Zugang quasi naturalisiert wird. Es muss aber im Sinne von Walter Benjamins ,Zur Kritik der Gewalt“ (Benjamin 1999 [1921]) die Frage gestellt werden, ob Gewalt überhaupt als Mittel zu gerechten Zwecken sittlich sein kann. Das Gewaltmonopol liegt dabei in der wissenschaftlichen (Sozial-)Pädagogik, die durch das, was hier als (Sozial-)Pädagogik zur Darstellung gebracht wird, (de)legitimiert wird. Das bedeutet, dass die wissenschaftliche (Sozial-)Pädagogik durch die Schulung zur höheren Sinnbildung ein Recht dazu hat, aber das bedeutet nicht unbedingt, dass das Urteil dadurch gerecht ist.

Gegenwärtig, d.h. im Kontext der Kinderrechte würde wohl dieses keine überzeugende Repräsentation eines sozialpädagogischen Falls sein, weil sich die gesellschaftlichen Normen hinsichtlich der Verletzung von Kinderrechten verändert haben, die das gleiche soziale Geschehen in einem anderen Licht erscheinen lassen. Das bedeutet aber, dass es notwendig ist, eine historische Kontingenz in der Bestimmung 
eines Falls als Fall von Sozialpädagogik mit zu berücksichtigen. Damit geht einher, dass die ,dichte Beschreibung ' des Falls für die Genese einer (sozial-)pädagogischen Tatsachenforschung von der heutigen Perspektive aus nicht als mögliche, sondern als unmögliche (Sozial-)Pädagogik betrachtet wird, es sich also um einen gescheiteren Fall von Sozialpädagogik handelt. Somit wäre es nicht mehr Vorbild, sondern Ausgangspunkt sozialpädagogischer Kritik.

\section{Schlussbemerkungen}

Mit A. Fischer konnte aufgezeigt werden, welche zentrale Bedeutung die Sozialwissenschaften für die Konstitution der Sozialpädagogik haben. Zugleich wurde aber auch deutlich, dass die Sozialpädagogik nicht in diesen aufgeht, sondern vielmehr, um den Ausdruck A. Fischers hinsichtlich der Soziologie der Erziehung aufzugreifen, eine „Zwischenwissenschaft“" bildet. Sie verändert ihre Gestalt, indem sie sich differenziell zu den anderen Sozialwissenschaften durch ,dichte Beschreibungen“ realisiert, d.h. verwirklicht (sozialpädagogischer Beruf) und sich darüber wissenschaftlich, d.h. durch ,dichte Beschreibungen ' aufklärt. Es entsteht dabei eine relativ eigenständige performative Kultur, zugleich ist sie in ihrer Erkenntnisbildung von den anderen sozialwissenschaftlichen Disziplinen durchdrungen. Die pädagogische Soziologie spielt dabei eine „Grenz- und Zwischenwissenschaft“ (Fischer 1954e [1932], S. 107), der die soziologische Aufklärung über die sozialpädagogischen Tatsachen obliegt.

Die sozialpädagogische Tatsache geht einerseits der wissenschaftlichen Sozialpädagogik voraus, anderseits ist sie aber durch den akademisch vermittelten sozialpädagogischen Berufsethos mit gestaltet. Die sozialpädagogische Tatsachenforschung wird zu einer reflexiven Rekonstruktion der Geschichte ihrer Habitualisierung, d.h. der Ausbildung des sozialpädagogischen Berufsethos, welcher zwischen den (sozialpädagogischen) Milieus der Handlungsfelder wie der sozialpädagogische Erkenntnisbildung durch, dichte " Beschreibungen vermittelt und zugleich miterzeugt wird. Dadurch transformiert sich die (sozial-)epistemiologische Perspektive auf Sozialpädagogik in eine genetische als eine sozialpädagogische Geschichte, die dialektisch zwischen soziologischer und sozialpsychologischer Aufklärung einerseits und sozialpädagogischer Tatsachen(forschung) andererseits vermittelt.

Der Vorwurf an A. Fischer das Proprium der sozialpädagogischen Tatsachenforschung an die Nachbardisziplin abgegeben zu haben (vgl. Reyer 2000; Niemeyer 1998) erscheint nicht als Problem, sondern als ihre eigene transdisziplinäre Möglichkeit sich einerseits relativ eigenständig, andererseits aber auch immer problemlösend auf die anderen Disziplinen beziehend zu konstituieren. Im Unterschied zum Professionalitätsdiskurs wird mit der Referenz auf Transdisziplinarität die Grenze der Sozialpädagogik als Disziplin kritisch in den Blick genommen, anstatt eine identifizierende Verbindung zwischen Disziplin und Profession zu suchen. Vielmehr ist von einem Bruch zwischen der sozialpädagogischen Intention als Berufsethos und ihrer Verwirklichung auszugehen. Dieser Bruch kann durch eine interdisziplinäre Forschung, ,die sich aus ihren disziplinären Grenzen löst, die ihre Probleme disziplinenunabhängig definiert und disziplinenunabhängig löst“ (Mittelstraß 1993, S. 26 ff., 
1996, S. 329), erforscht werden. Im Unterschied zu den ,klassischen Professionen“ weiß die transdisziplinäre Sozialpädagogik um ihre bedingte Möglichkeit, sozialpädagogische Tatsachen im doppelten Sinne zu realisieren Das bedeutet, dass das Nicht-Gelingen als Möglichkeit der sozialpädagogischen Tat mitgedacht wird. Dann handelt es sich um eine Kraft, der eine ,praktische Unmöglichkeit“ (Menke 2013, S. 24) zugrunde liegt, die sie paradoxerweise zugleich erst möglich macht. Diese Kraft basiert auf einem Machen, sonst wäre sie nicht praktisch, aber sie basiert nicht auf einer wissbaren Erkenntnis, sonst wäre der Fall in Relation zu dem Zusammenhang der vorausgehenden Fälle nicht neu. Das Neue in dem Fall, die Vermittlung zwischen der praktischen Hervorbringung unter der Grenze der wissensbezogenen Erkenntnis, bedeutet, sich selbst in seinem Vermögen der Erkenntnisgenerierung zu bekämpfen (vgl. Menke 2013, S. 29). Dadurch wird die Spannung zwischen intuitiver und gestaltschließender Erkenntnis aufrechterhalten (vgl. Badura 2015, S. 46). Das bedeutet aber auch für das Verständnis von Intervention, dass es ein inter-venire ist, etwas das zwischen theoretischer Erkenntnis der Sozialpädagogik und der Praxis im Kommen gehalten wird und damit die Möglichkeitsdimension gegenüber der Wirklichkeitsdimension begründet wird. Dieses vollzieht sich aber einerseits über sinnliche Wahrnehmung und andererseits über deren Erkenntnisform einer Darstellungsform, in der gezeigt wird, wie man von der Vulnerabilität der (Re-)Präsentation der sozialpädagogischen Praxis, nicht das sein zu können, was sie vorgibt zu sein, berührt ist, um sich im Horizont gegebener Möglichkeit als andere Erkenntnisform zu verwirklichen.

In der Diskursgeschichte der Sozialpädagogik kann die sozial(pädagogisch)e Wissensproduktion zum Gegenstand gemacht werden. Dabei wird davon ausgegangen, dass die gleiche sozialpädagogische Tatsache in einem anderen historischen Kontext eine andere ist, wodurch die „Struktur der [Sozial-]Pädagogik“ (Koller 2012, S. 23) in ein prozessuales Geschehen transformiert wird (Koller 2012, S. 18). Sie kann nicht „festgestellt“, sondern als Sinnzusammenhang nur differenziell in historisch dialektischer Bewegung entwickelt werden. Dadurch entsteht die „,kritische und tragische Erkenntnis“, dass die „Selbständigkeit des Gegenstandes gegenüber dem Erkennen und Handeln der Subjekte zu verneinen sei“ (Lütke-Harmann 2019, S. 271). Das setzt eine notwendige Differenz zwischen Repräsentierten und der Repräsentation voraus (vgl. Lütke-Harmann 2019, S. 274). Aber auch diese Annahme kann nicht als „festes Fundament“ gesetzt werden, sondern vielmehr gehe es darum, ihre Grundlosigkeit und damit zugleich auch ihre Scheinhaftigkeit in die Reflexion aufzunehmen“ (Lütke-Harmann 2019, S. 275). Es ginge darum, einen „Modus des Denkens“ einzunehmen, der auf die Unabschließbarkeit der Erkenntnis und damit auf ihre Historizität beruhe (Lütke-Harmann, S. 275). Während die Theorie der Sozialpädagogik die Formen der Instituierung des Sozialpädagogischen in einer Fallgeschichte innerhalb einer vorausgesetzten Formation benennt, steht die kritische Diskursgeschichte der Sozialpädagogik für einen „Vorgang, innerhalb dessen diese Ordnung und ihre Repräsentation selbst zu Disposition gestellt werden“ (Lütke-Harmann 2019, S. 277). Dadurch kann sich die Sozialpädagogik auf einen Möglichkeitshorizont hin aufzeigend öffnen, eben transformativ werden.

Funding Open Access funding provided by Projekt DEAL. 
Open Access Dieser Artikel wird unter der Creative Commons Namensnennung 4.0 International Lizenz veröffentlicht, welche die Nutzung, Vervielfältigung, Bearbeitung, Verbreitung und Wiedergabe in jeglichem Medium und Format erlaubt, sofern Sie den/die ursprünglichen Autor(en) und die Quelle ordnungsgemäß nennen, einen Link zur Creative Commons Lizenz beifügen und angeben, ob Änderungen vorgenommen wurden.

Die in diesem Artikel enthaltenen Bilder und sonstiges Drittmaterial unterliegen ebenfalls der genannten Creative Commons Lizenz, sofern sich aus der Abbildungslegende nichts anderes ergibt. Sofern das betreffende Material nicht unter der genannten Creative Commons Lizenz steht und die betreffende Handlung nicht nach gesetzlichen Vorschriften erlaubt ist, ist für die oben aufgeführten Weiterverwendungen des Materials die Einwilligung des jeweiligen Rechteinhabers einzuholen.

Weitere Details zur Lizenz entnehmen Sie bitte der Lizenzinformation auf http://creativecommons.org/ licenses/by/4.0/deed.de.

\section{Literatur}

\section{Primärliteratur}

Badura, J. (2015). Erkenntnis (sinnliche). In J. Badura, S. Dubach, A. Haarmann, D. Mersch, A. Rey, C. Schenker \& G. T. Pérez (Hrsg.), Künstlerische Forschung. Ein Handbuch (S. 43-48). Zürich: Diaphanes.

Benjamin, W. (1921/1999). Zur Kritik der Gewalt. In W. Benjamin (Hrsg.), Gesammelte Schriften (Bd. II, S. 179-204). Frankfurt: Suhrkamp.

Bonß, W. (1983). Kritische Theorie als empirische Wissenschaft. Zur Methodologie ,postkonventioneller Sozialforschung. Soziale Welt, 34(1), 57-89.

Brinkmann, M. (2017). Phänomenologische Erziehungswissenschaft. Ein systematischer Überblick von ihren Anfängen bis heute. In M. Brinkmann, S. Rödel \& M. F. Buck (Hrsg.), Pädagogik - Phänomenologie. Phänomenologie - Pädagogik. Verhältnisbestimmungen und Herausforderungen (S. 17-46). Wiesbaden: Springer.

Cloos, P., Lochner, B., \& Schoneville, H. (2020). Soziale Arbeit als Projekt. In P. Cloos, B. Lochner \& H. Schoneville (Hrsg.), Soziale Arbeit als Projekt (S. 1-9). Wiesbaden: Springer VS.

Dollinger, B. (2006). Die Pädagogik der Sozialen Frage. (Sozial-)pädagogische Theorie vom Beginn des 19. Jahrhunderts bis zum Ende der Weimarer Republik (1. Aufl.). Wiesbaden: VS.

Fischer, A. (1950). Deskriptive Pädagogik. Die Lage der Psychologie in der Gegenwart und ihre Folgen für die psychologische Jugendforschung. In K. Kreitmair (Hrsg.), Aloys Fischer. Leben und Werk (Bd. 1, S. 5-30). München: Bayrischer Schulbuchverlag.

Fischer, A. (1954a). Soziologie und Sozialwissenschaften, Sozialpsychologie. In K. Kreitmair (Hrsg.), Aloys Fischer. Leben und Werk (Bd. 3 und 4, S. 25-68). München: Bayrischer Schulbuchverlag. 1923.

Fischer, A. (1954b). Das Verhältnis der Jugend zu den sozialen Bewegungen und der Begriff der Sozialpädagogik. In K. Kreitmair (Hrsg.), Aloys Fischer. Leben und Werk (Bd. 3 und 4, S. 167-262). München: Bayrischer Schulbuchverlag. 1924.

Fischer, A. (1954c). Die Problematik des Sozialbeamtentums. In K. Kreitmair (Hrsg.), Aloys Fischer. Leben und Werk (Bd. 3 und 4, S. 319-350). München: Bayrischer Schulbuchverlag. 1925.

Fischer, A. (1954d). Soziologische Pädagogik. In K. Kreitmair (Hrsg.), Aloys Fischer. Leben und Werk (Bd. 3 und 4, S. 159-167). München: Bayrischer Schulbuchverlag. 1932.

Fischer, A. (1954e). Pädagogische Soziologie. In K. Kreitmair (Hrsg.), Aloys Fischer. Leben und Werk (Bd. 3 und 4, S. 107-158). München: Bayrischer Schulbuchverlag. 1932.

Fischer, A. (1957a). Untergründe und Hintergründe des Bewußtseins (Eine Skizze des Problems des Unbewußten in der heutigen Psychologie). In: K. Kreitmair (Hrsg.), Aloys Fischer. Leben und Werk (Bd. 5 und 6, S. 57-134). München. Bayrischer Schulbuchverlag. 1915.

Fischer, A. (1957b). Die Lage der Psychologie in der Gegenwart und ihre Folgen für die psychologische Jugendforschung. In K. Kreitmair (Hrsg.), Aloys Fischer. Leben und Werk (Bd. 5 und 6, S. 31-56). München: Bayrischer Schulbuchverlag. 1925. 
Fischer, A. (1957c). Untergründe und Hintergründe des Bewußtseins (Eine Psychologie des Problems des Unbewußten in der heutigen Psychologie). In K. Kreitmair (Hrsg.), Aloys Fischer. Leben und Werk (Bd. 5 und 6, S. 57-112). München: Bayrischer Schulbuchverlag. 1925.

Fischer, A. (1957d). Der Lehrer als pädagogischer Berufstyp. In K. Kreitmair (Hrsg.), Fischer, Aloys. Leben und Werk (Bd. 5 und 6, S. 303-354). München: Bayrischer Schulbuchverlag. 1926.

Fischer, A. (1967a). Erziehungsfürsorge. In K. Kreitmair (Hrsg.), Aloys Fischer. Leben und Werk (Bd. 7, S. 459-470). München: Bayrischer Schulbuchverlag.

Fischer, A. (1967b). Beruf und Berufserziehung. In K. Kreitmair (Hrsg.), Aloys Fischer. Leben und Werk (Bd. 7, S. 441-458). München: Bayrischer Schulbuchverlag. 1932.

Flösser, G., \& Wohlgemuth, K. (2011). Soziale Kontrolle. In H.-U. Otto \& H. Thiersch (Hrsg.), Handbuch Soziale Arbeit (S. 1365-1375). München: Ernst Reinhardt.

Geertz, C. (1987). Dichte Beschreibung. Frankfurt am Main: Suhrkamp.

Gundlach, H., \& Stöver, R. (2004). Die Gesellschaft für experimentelle Psychologie, später Deutsche Gesellschaft für Psychologie und ihre Kongresse 1904 bis 1932. Psychologische Rundschau, 55(1), $12-20$.

Heinemann, R. (2016). Das Kind als Person. William Stern als Wegbereiter der Kinder- und Jugendforschung 1900 bis 1933. Bad Heilbrunn: Klinkhardt.

Herzog, M. (1992). Phänomomenologische Psychologie. Grundlagen und Entwicklungen. Heidelberg: Asanger.

Kessl, F. (2020). Von der BeGründung Sozialer Arbeit. In P. Cloos, B. Lochner \& H. Schoneville (Hrsg.), Soziale Arbeit als Projekt (S. 85-94). Wiesbaden: Springer VS.

Kessl, F., \& Maurer, S. (2010). Praktiken der Differenzierung als Praktiken der Grenzbearbeitung. Überlegungen zur Bestimmung Sozialer Arbeit als Grenzbearbeiterin. In F. Kessl \& M. Plößer (Hrsg.), Differenzierung, Normalisierung, Andersheit (S. 154-169). Wiesbaden: VS.

Kohlberg, L. (1984). Der „Just Community“-Ansatz der Moralerziehung in Theorie und Praxis. In Oser, F., Fatke, R. \& Höffe, O. (Hrsg.), Transformation und Entwicklung: Grundlagen der Moralerziehung. (S. 21-55). Frankfurt a.M.: Suhrkamp.

Koller, C. (2012). Bildung anders denken. Einführung in die Theorie transformatorischer Bildungsprozesse. Stuttgart: Kohlhammer.

Korte, H. (2004). Einführung in die Geschichte der Soziologie (7. Aufl.). Opladen: Leske + Budrich.

Kreitmair, K. (1954). Aloys Fischers Entwicklung zum Soziologen. In K. Kreitmair (Hrsg.), Aloys Fischer. Leben und Werk (Bd. 3 und 4, S. 9-24). München: Bayrischer Schulbuchverlag.

Kruse, J. (2009). Indexikalität und Fremdverstehen: Problemfelder kommunikativer Verstehensprozesse. In B. Rehbein \& G. Saalmann (Hrsg.), Verstehen (S. 133-150). Konstanz: UVK.

Lepsius, M. R. (2011). Max Weber und die Gründung der Deutschen Gesellschaft für Soziologie. Soziologie, 40(1), 7-19.

Lippitz, W. (2020). Aloys Fischer (1880-1937): „Deskriptive Pädagogik oder Prinzipienwissenschaft von der Erziehung“. Zu den Anfängen phänomenologischer Forschungen in der Erziehungswissenschaft. In I.W. Lippitz \& M. Brinkmann (Hrsg.), Phänomene der Erziehung und Bildung. Phänomenologisch-Pädagogische Studien (S. 155-173). Wiesbaden: Springer VS.

Lüders, C. (1989). Der wissenschaftlich ausgebildete Praktiker. Entstehung und Auswirkung des TheoirePraxis-Konzeptes des Diplomstudienganges Sozialpädagogik. Weinheim: Deutscher Studienverlag.

Lütke-Harmann, M. (2016). Symbolische Metamorphosen. Eine problemgeschichtliche Studie zur politischen Epistemologie der Sozialpädagogik (1. Aufl.). Weilerswist: Velbrück Wissenschaft.

Lütke-Harmann, M. (2019). Sozialpädagogische Theorie als Politische Theorie des Sozialen. Zeitschrift für Sozialpädagogik, 17(3), 266-281.

Menke, C. (2013). Die Kraft der Kunst. Frankfurt am Main: Suhrkamp.

Mittelstraß, J. (1993). Interdisziplinarität oder Transdisziplinarität? In L. Hieber (Hrsg.), Utopie Wissenschaft. Ein Symposium an der Universität Hannover über die Chancen des Wissenschaftsbetriebs der Zukunft (21./22. November 1991). Reihe Wissenschaft. (S. 17-31). München: Profil.

Mittelstraß, J. (1996). Transdisziplinarität. In J. Mittelstraße (Hrsg.), Enzyklopädie Philosophie und Wissenschaftstheorie Bd. 4. Stuttgart und Weimar: Metzler.

Münchmeier, R. (2004). Zwischen Pädagogik und Infrastruktur. Zum wissenschaftlichen Programm der Sozialpädagogik. In R. Hörster, E.-U. Küster \& S. Wolff (Hrsg.), Orte der Verständigung. Beiträge zum sozialpädagogischen Argumentieren. Burkhard Müller zum 65. Geburtstag gewidmet (S. 21-34). Freiburg im Breisgau: Lambertus.

Neumann, S. (2008). Kritik der sozialpädagogischen Vernunft. Feldtheoretische Studien (1. Aufl.). Weilerswist: Velbrück.

Niemeyer, C. (1998). Klassiker der Sozialpädagogik. Weinheim: Juventa. 
Renn, J. (2014). Performative Kultur und Multiple Differenzierung. Bielefeld: transcript.

Reyer, J. (2000). Individualpädagogik und Sozialpädagogik - Eine Skizze zur Entwicklung sozialpädagogischer Denkformen. In J. Henseler \& J. Reyer (Hrsg.), Sozialpädagogik und Gemeinschaft (S. 23-39). Baltmannsweiler: Schneider-Verlag Hohengehren.

Reyer, J. (2002). Kleine Geschichte der Sozialpädagogik. Individuum und Gemeinschaft in der Pädagogik der Moderne. Baltmannsweiler: Schneider-Verlag Hohengehren.

Röhrs, H. (1967). Die Pädagogik Aloys Fischers. Heidelberg: Quelle \& Meyer.

Sala, R. (2017). The Rise of Sociology. Geschichte und Gesellschaft, 43(4), 557-584.

Sandermann, P., \& Neumann, S. (2018). Grundkurs Theorien der Sozialen Arbeit. Stuttgart: UTB.

Simmel, G. (1920). Grundlagen der Soziologie. Berlin: Duncker \& Humblot.

Simmel, G. (1983). Untersuchungen über die Formen der Vergesellschaftung. Berlin: Duncker \& Humblot. 1908

Stadler-Altmann, U., \& Gross, B. (2019). Beyond erziehungswissenschaftlicher Theorie und Praxis: Einführung in den Thementeil. In U. Stadler-Altmann \& B. Gross (Hrsg.), Beyond erziehungswissenschaftlicher Grenzen. Diskurse zu Entgrenzungen der Disziplin (1. Aufl. S. 9-16). Leverkusen: Verlag Barbara Budrich.

Stephani, H. (1805). Das System der öffentlichen Erziehung. Berlin: Föhlich.

Stichweh, R. (2017). Interdisziplinarität und wissenschaftliche Bildung. In H. Kauhaus \& N. Krause (Hrsg.), Fundiert forschen (S. 181-190). Wiesbaden: Springer VS.

Thole, W. (1994). Sozialpädagogik an zwei Orten: Professionelle und disziplinäre Ambivalenzen eines noch unentschiedenen Projektes. In H.-H. Krüger \& T. Rauschenbach (Hrsg.), Erziehungswissenschaft (S. 253-274). Weinheim: Juventa.

Thole, W., \& Galuske, M. (2003). Sozialpädagogik - „Jahrhundertprojekt“ oder „Entsorgungsfall“? Zeitschrift für Pädagogik, 49(6), 885-902.

Weber, M. (1919). Wissenschaft als Beruf (S. 33). München und Leipzig: Duncker \& Humblot. Digitale Volltext-Ausgabe bei Wikisource. https://de.wikisource.org/w/index.php?title=Seite:Max_Weber_-_ Wissenschaft_als_Beruf_-_Seite_33.jpg\&oldid=- (Version vom 1.8.2018)

Wendt, A. N. (2019). Phänomenologische Psychologie. In G. Mey \& K. Mruck (Hrsg.), Handbuch Qualitative Forschung in der Psychologie (Bd. 14, S. 1-24). Wiesbaden: Springer VS.

Winkler, M. (1988). Eine Theorie der Sozialpädagogik. Stuttgart: Klett-Cotta.

Wolff, S. (1992). Die Anatomie der Dichten Beschreibung. Soziale Welt, Sonderband 8, 339-362.

\section{Weiterführende Literatur}

Fischer, A. (1967c). Über die Bedeutung des Experiments in der pädagogischen Forschung die Idee einer exakten Pädagogik. In H. Röhrs (Hrsg.), Erziehungswissenschaft und Erziehungswirklichkeit (S. 35-57). Frankfurt am Main: Akademische Verlagsgesellschaft. 1913. 